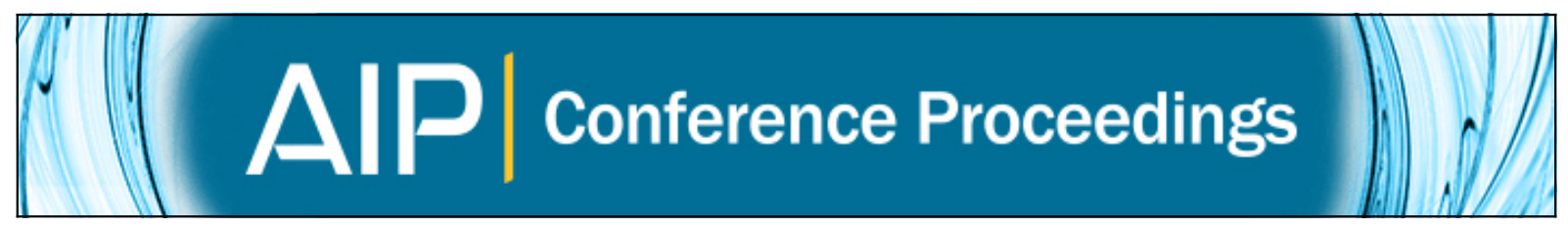

\title{
A new approach to the cosmological moduli problem
}

Keith R. Dienes, Jeff Kost, and Brooks Thomas

Citation: AIP Conference Proceedings 1743, 020003 (2016); doi: 10.1063/1.4953270

View online: http://dx.doi.org/10.1063/1.4953270

View Table of Contents: http://scitation.aip.org/content/aip/proceeding/aipcp/1743?ver=pdfcov

Published by the AIP Publishing

Articles you may be interested in

A New Approach to the Coronal Heating Problem

AIP Conf. Proc. 1188, 153 (2009); 10.1063/1.3266793

A new perturbative approach to nonlinear problems

J. Math. Phys. 30, 1447 (1989); 10.1063/1.528326

A new approach to inhomogeneous cosmologies: Intrinsic symmetries. I

J. Math. Phys. 20, 2347 (1979); 10.1063/1.524017

A new approach to the inverse problem

J. Math. Phys. 19, 2619 (1978); 10.1063/1.523617

New Approach to the Ising Problem

J. Math. Phys. 7, 305 (1966); 10.1063/1.1704933 


\title{
A New Approach to the Cosmological Moduli Problem
}

\author{
Keith R. Dienes ${ }^{1,2, a)}$, Jeff Kost ${ }^{1, b)}$ and Brooks Thomas ${ }^{3,4, c)}$ \\ ${ }^{1}$ Department of Physics, University of Arizona, Tucson, AZ 85721 USA \\ ${ }^{2}$ Department of Physics, University of Maryland, College Park, MD 20742 USA \\ ${ }^{3}$ Department of Physics, Reed College, Portland, OR 97202 USA \\ ${ }^{4}$ Department of Physics, Colorado College, Colorado Springs, CO 80903 USA \\ a)dienes@email.arizona.edu \\ b)jkost@email.arizona.edu \\ c)bthomas@coloradocollege.edu
}

\begin{abstract}
A generic byproduct of many theories beyond the Standard Model is the appearance of light scalar fields known as moduli. These moduli should be copiously produced in the early universe but have dangerously long lifetimes, leading to their excessive domination of the late-time energy density — an issue known as the "cosmological moduli problem". In this talk, we discuss a number of new effects which have direct relevance for the cosmological moduli problem and which, depending on circumstances, can either unexpectedly amerliorate it or worsen it, often by many orders of magnitude. As described more fully in Ref. [1], these effects arise in theories containing multiple moduli which mix amongst themselves in the presence of a massgenerating phase transition.
\end{abstract}

\section{INTRODUCTION}

One generic property of string theories is that their four-dimensional vacua are characterized by a set of continuous parameters, or moduli, that serve to parametrize the compactification. These moduli are scalar fields with a potential that is flat in all directions of field space, making them massless. Such flat potentials lead to severe problems, including the fact that the eventual vacuum expectation values (VEVs) of such fields are completely undetermined. A mechanism is thus required by which the potential for the moduli can acquire a positive curvature, thereby giving a mass to (and thereby stabilizing) the moduli.

At first glance, it might appear this is actually a non-issue and that the moduli might be stabilized by quantum loop corrections. The expected mass scale for these corrections would be at the scale of supersymmetry breaking, and thus the resulting moduli masses $m_{\phi}$ would not exceed the gravitino mass. The latter, in turn, is bounded above by $\lesssim 1 \mathrm{TeV}$ in order to ensure a natural solution to the hierarchy problem. However, this scenario produces several cosmological problems. The moduli, which would have initial vacuum values of order the Planck mass $M_{P}$, would therefore dominate the energy density of the universe unless they decayed sufficiently prior to Big Bang Nucleosynthesis. This requires that the moduli decay before the universe cools to a temperature $\sim 1 \mathrm{MeV}$. However, the fact that the decay rate for the moduli could be at most $O\left(m_{\phi}^{3} / M_{P}^{2}\right)$ requires masses $m_{\phi}$ that exceed the TeV scale, thus requiring a supersymmetry-breaking scale that is disturbingly large. These difficulties are collectively dubbed the "cosmological moduli problem". Indeed, this problem occurs not only in string models [2, 3, 4] but also in supergravity [5], where it was first encountered.

The time-dependence of the effective moduli potential has been discussed in the past [6] using toy models and been found to lead to a suppressed moduli energy density. Within an axion context, it has similarly been demonstrated that the total energy density can be suppressed in multi-axion models when mixing between the fields is generated along with their masses $[7,8,9]$ or via some other mechanism [10]. Nevertheless, the effects of mixing and the effects of a time dependence in mass-generating phase transitions have always typically been studied in isolation.

In a recent paper [1] it was shown that when both effects are considered simultaneously, a number of surprising new phenomena can emerge. These results were obtained within the context of a generic scalar toy model. However, when interpreted in the context of moduli, these features could significantly impact the cosmological moduli problem; 
indeed, depending on the parameter configuration of the given model, these results could change the predicted latetime moduli energy densities by many orders of magnitude! In this talk, we shall quickly survey these findings. Further details can be found in Ref. [1].

\section{A TOY MODEL}

In this section we delineate the toy model which shall be the basis of our analysis. As we shall see, our toy model is simple enough to be tractable, yet rich enough to incorporate all of the phenomena of interest. Our toy model contains two moduli $\phi_{0}$ and $\phi_{1}$. Recall that in a flat FRW universe, a spatially-constant scalar field $\phi$ with time-dependent mass $m_{\phi}(t)$ evolves according to the equation of motion

$$
\frac{\partial^{2} \phi}{\partial t^{2}}+3 H(t) \frac{\partial \phi}{\partial t}+m_{\phi}^{2}(t) \phi=0
$$

where the Hubble parameter $H(t)$ scales as $3 H(t) \approx \kappa / t$ with $\kappa=2$ (respectively $\kappa=3 / 2$ ) within a matter- (radiation-) dominated epoch. In other words, $\phi(t)$ evolves as a damped harmonic oscillator, with time-dependent damping parameter $\zeta(t) \equiv 3 H /(2 m)$ and mass $m_{\phi}$. Since $H(t)$ falls with time, an initially overdamped moduli field will inevitably become underdamped and undergo decaying oscillations. The energy density $\rho$ associated with such a field will thus initially behave as vacuum energy and at the time of critical damping $t_{\zeta}$ transition to a phase where it behaves as massive matter. This evolution is shown for a constant-mass field in Fig. 1. Note that we do not consider phase transitions occurring during the inflation epoch, and it is assumed that the back-reaction of the moduli on the rate $H(t)$ is negligible.

For two fields $\phi_{0}$ and $\phi_{1}$, we imagine that our mass term is generated by a phase transition which not only introduces effective masses for our fields but also introduces a mixing between them:

$$
\frac{\partial^{2} \phi_{i}}{\partial t^{2}}+3 H(t) \frac{\partial \phi_{i}}{\partial t}+\sum_{j} \mathcal{M}_{i j}^{2}(t) \phi_{j}=0
$$

where $\mathcal{M}^{2}$ is the corresponding squared-mass matrix. At times $t \ll t_{G}$, long before mass generation occurs, we shall take $\mathcal{M}^{2}$ as constant and diagonal: $\mathcal{M}^{2}=\operatorname{diag}\left(0, M^{2}\right)$ where $M \neq 0$ is a general unfixed mass parameter. By contrast, long after the phase transition, we assume that additional mass-matrix components $\bar{m}_{i j}^{2}$ have been generated. In order to connect these two asymptotic extremes, we shall let $m_{i j}^{2}(t)$ denote time-dependent elements of the mass matrix which interpolate between zero at early times and $\bar{m}_{i j}^{2}$ at late times. We can then explicitly write the squared-mass matrix as

$$
\mathcal{M}^{2}(t)=\left[\begin{array}{cc}
0 & 0 \\
0 & M^{2}
\end{array}\right]+\left[\begin{array}{cc}
m_{00}^{2}(t) & m_{01}^{2}(t) \\
m_{01}^{2}(t) & m_{11}^{2}(t)
\end{array}\right] .
$$

Having specified the mass matrix, we now introduce a dimensionless time variable $\tau \equiv M t$ which scales away the mass $M$. With this change of variables our mass matrix is dimensionless and each $m_{i j}$ is now understood implicitly to be written as a fraction of $M$. We shall further make the simplifying assumption that the time-dependence of each component in $\mathcal{M}^{2}$ is identical, as would arise from a single phase transition with a single associated timescale. Indeed, since the mass matrix is nothing but the curvature matrix associated with the moduli potential $V\left(\phi_{0}, \phi_{1}\right)$ induced by the phase transition, this assumption is tantamount to assuming a single time-dependence for the potential as a whole. As a result, we can write each of the individual mass components in a factorized form

$$
m_{i j}(\tau)=\bar{m}_{i j} \cdot h(\tau),
$$

where $h(\tau)$ is a smooth function of time which describes the time-development of the phase transition. We require that $h(\tau) \rightarrow 0$ as $\tau \rightarrow 0$ and $h(\tau) \rightarrow 1$ as $\tau \rightarrow \infty$. We would also like $h(\tau)$ to also incorporate a dimensionless parameter $\sigma$ which controls how abruptly the transition occurs. Additionally, it is desirable that the midpoint of the transition $\tau_{G}$ be a fixed point of reference whenever $\sigma$ is varied. Putting these requirements together, we shall make the choice

$$
h(\tau)=\frac{1}{2}\left\{1+\operatorname{erf}\left[\frac{1}{\sigma} \log \left(\frac{\tau}{\tau_{G}}\right)\right]\right\} .
$$



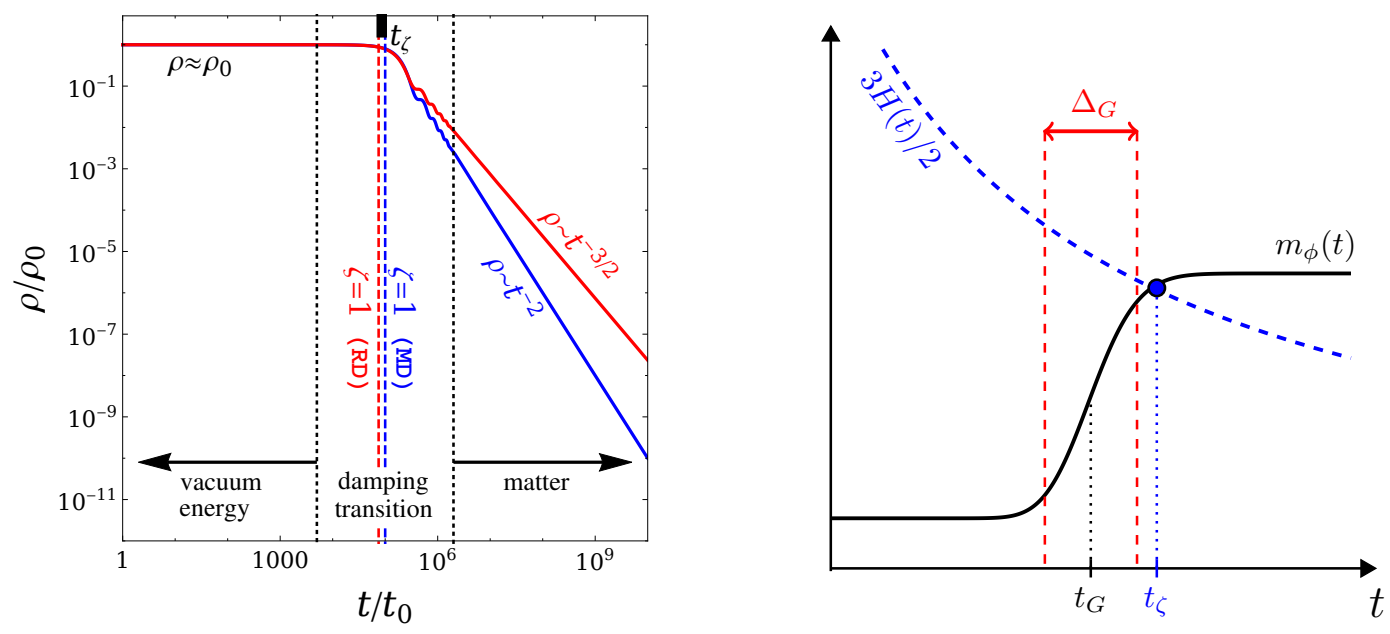

FIGURE 1. Left panel: the energy density $\rho$, normalized to its initial value $\rho_{0}$, for a solution to Eq. (1) in a radiation-dominated (RD) universe, i.e., $\kappa=3 / 2$ (red), or a matter-dominated (MD) universe, i.e., $\kappa=2$ (blue). In the overdamped phase, the energy density remains nearly constant at $\rho=-p>0$, behaving as vacuum energy. By contrast, once the critical damping $\zeta=1$ threshold is crossed at $\tau_{\zeta}$, the energy density begins to dissipate, asymptotically exhibiting the simple power-law scaling behavior $\rho \sim \tau^{-\kappa}$ expected for matter. Right panel: a schematic of the time-dependence of a single mass component, with the Hubble damping curve $3 H / m_{\phi}$ superimposed and the width of the transition $\Delta_{G}$ and the critical damping time $t_{\zeta}$ marked.

Corresponding to each non-zero value of the parameter $\sigma$ there exists a non-zero timescale

$$
\Delta_{G} \equiv \sqrt{\pi} \sigma \tau_{G}
$$

over which the phase transition occurs. This "width" of the phase transition will be of central importance in our analysis. We note that $\Delta_{G} \rightarrow 0$ as $\sigma \rightarrow 0$; this limit corresponds to the case of an instantaneous phase transition, with $h(\tau)$ taking the form of the Heaviside step function $\Theta\left(\tau-\tau_{G}\right)$. As $\sigma$ is increased, the width of the transition $\Delta_{G}$ increases until $\sigma=\sqrt{2}$, beyond which our $h(\tau)$ function no longer describes mass generation at $\tau_{G}$. We therefore impose this value of $\sigma$ as an effective upper-bound on the abruptness of the transition.

The only remaining ingredient to be specified as part of our toy model is a set of initial conditions to be imposed at some initial time $\tau_{0} \ll \tau_{G}$. The natural and well-motivated choice in the context of moduli is a mere displacement of the $\phi_{0}$ field at some initial time $\tau_{0}$ :

$$
\left(\begin{array}{c}
\phi_{0} \\
\phi_{1}
\end{array}\right)_{\tau=\tau_{0}}=\left(\begin{array}{c}
A_{0} \\
0
\end{array}\right), \quad\left(\begin{array}{c}
\dot{\phi}_{0} \\
\dot{\phi}_{1}
\end{array}\right)_{\tau=\tau_{0}}=\left(\begin{array}{l}
0 \\
0
\end{array}\right),
$$

where $A_{0}$ serves to set an overall mass scale for our system. This scale will typically be set by $M_{P}$ in the context of string moduli, but since we shall always be considering ratios of field values or energy densities, our results will be insensitive to this scale and we shall never set it explicitly.

It will prove useful to first introduce the variables

$$
\begin{aligned}
m_{\text {sum }}^{2} & \equiv \mathcal{M}_{11}^{2}+\mathcal{M}_{00}^{2}=1+m_{00}^{2}+m_{11}^{2} \\
\Delta m^{2} & \equiv \mathcal{M}_{11}^{2}-\mathcal{M}_{00}^{2}=1-m_{00}^{2}+m_{11}^{2} .
\end{aligned}
$$

The only requirement we impose on $\mathcal{M}^{2}$ is that it produce real and non-negative eigenvalues, which is tantamount to requiring it be positive-semidefinite. This ultimately imposes a bound on the diagonal elements $\left|\Delta m^{2}\right| \leq m_{\text {sum }}^{2}$ and the off-diagonal element:

$$
0 \leq m_{01}^{2} \leq\left[m_{01}^{2}\right]_{\max }=\frac{1}{2} \sqrt{\left(m_{\text {sum }}^{2}\right)^{2}-\left(\Delta m^{2}\right)^{2}} .
$$

Furthermore, we parametrize the mixing in our toy model in terms of a rotation angle $\theta$ given by

$$
\tan (2 \theta)=\frac{2 m_{01}^{2}}{\Delta m^{2}}
$$


which relates the mass eigenstates $\phi_{\lambda_{0}}$ and $\phi_{\lambda_{1}}$ at any instant of time to $\phi_{0}$ and $\phi_{1}$. While any range of angles $\theta$ is certainly possible, only angles in the range $\theta \in\left[0, \frac{\pi}{2}\right]$ are required - any choice of mixing angle outside this range is in fact redundant. Note that the upper bound on $m_{01}^{2}$ in Eq. (9) clearly implies bounds on the mixing angle $\theta$ as well. We will often find that the crucial quantity is not the absolute degree of mixing, but the degree of mixing relative to this maximum value. We therefore typically specify configurations not by $m_{01}^{2}$ or $\theta$, but by the mixing saturation $0 \leq \xi \leq 1$ defined as

$$
\xi \equiv \frac{m_{01}^{2}}{\left[m_{01}^{2}\right]_{\max }}=\frac{\tan (2 \theta)}{\tan \left(2 \theta_{\max }\right)}=\frac{m_{01}^{2}}{\sqrt{m_{00}^{2}\left(1+m_{11}^{2}\right)}},
$$

where $\theta_{\max }$ as the angle corresponding to $\left[m_{01}^{2}\right]_{\max }$. An analogous observation can be made regarding the $\left|\Delta m^{2}\right| \leq m_{\mathrm{sum}}^{2}$ bound, and we therefore also define the splitting saturation:

$$
\eta \equiv \frac{\Delta m^{2}}{m_{\mathrm{sum}}^{2}}=\frac{1-m_{00}^{2}+m_{11}^{2}}{1+m_{00}^{2}+m_{11}^{2}}
$$

where $-1 \leq \eta \leq 1$.

Finally, during the mass-generating phase transition, almost all of the quantities discussed above are implicitly functions of time $\tau$ during the transition. For any such quantity $X(\tau)$ we define the notation

$$
\bar{X} \equiv \lim _{\tau \rightarrow \infty} X(\tau) .
$$

In other words, $\bar{X}$ shall denote the asymptotic late-time value of $X(\tau)$, where in practical terms the notion of a "late" time can be taken as referring to a time at which both moduli $\phi_{\lambda}$ have reached the asymptotic underdamped regime.

The time-dependence of the mass eigenvalues during the phase transition will be important for our future results. At any moment in time, the masses of the moduli are given by

$$
\lambda_{0,1}^{2}=\frac{1}{2} m_{\text {sum }}^{2}\left[1 \mp \sqrt{\eta^{2}+\left(1-\eta^{2}\right) \xi^{2}}\right]=\frac{1}{2} m_{\text {sum }}^{2}(1 \mp \eta \sec 2 \theta) .
$$

We show the evolution of these masses from early to late times in Fig. 2. Numerous features are immediately apparent. First, as predicted from Eq. (14), we see that the eigenvalues at early and late times ( $\tau \ll \tau_{G}$ and $\tau \gg \tau_{G}$, respectively) are independent of the sign of $\Delta m^{2}$ (or $\eta$ ). Thus, the early- and late-time values of $\lambda_{0,1}^{2}$ are identical in the left and right panels of Fig. 2. However, we see that the time-evolution of the eigenvalues between these two endpoints is highly
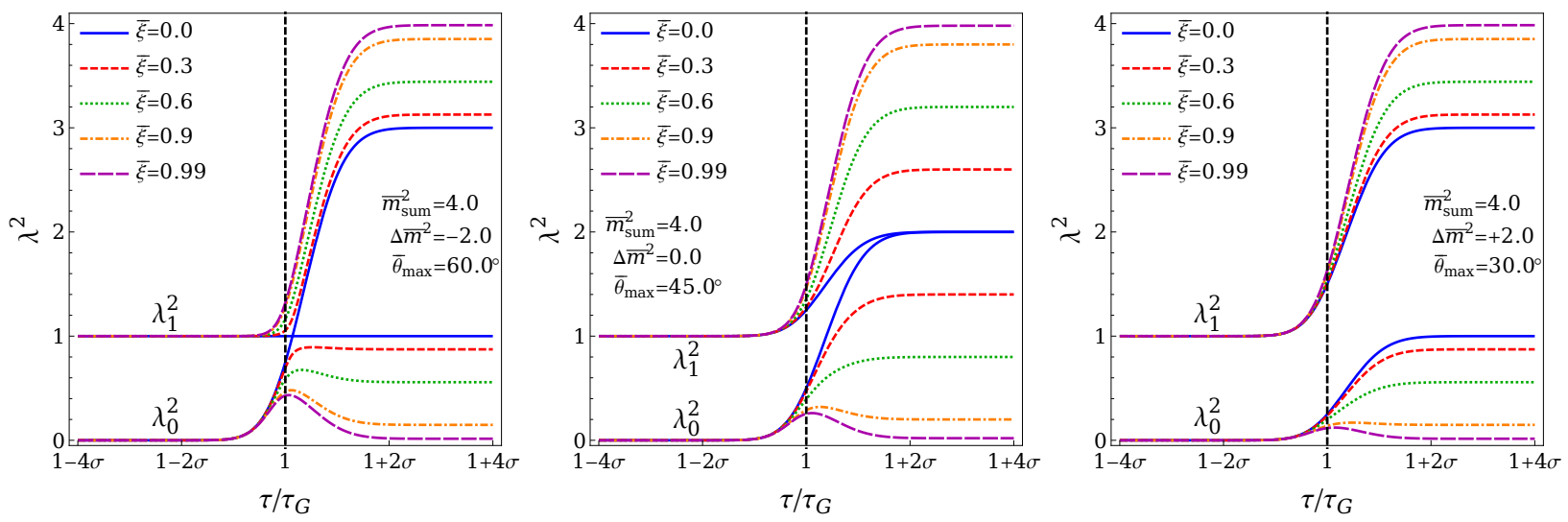

FIGURE 2. The time-evolution of the two mass eigenvalues $\lambda_{0,1}^{2}$ as the phase transition unfolds, plotted for different mixing saturations. Typical behaviors are shown for $\Delta \bar{m}^{2}<0$ (left panel), $\Delta \bar{m}^{2}=0$ (middle panel), and $\Delta \bar{m}^{2}>0$ (right panel). In all cases, the eigenvalues begin at $\{0,1\}$, as expected. For $\Delta \bar{m}^{2}>0$, the eigenvalues slowly transition to their late-time values without any tendency towards level-crossing. For $\Delta \bar{m}^{2}<0$, by contrast, the eigenvalues meet and rebound off each other in the case of zero mixing $(\bar{\xi}=0)$ but such a meeting is thwarted by level repulsion for all non-zero mixing. 
sensitive to the sign of $\Delta \bar{m}^{2}$. For $\Delta \bar{m}^{2}>0$, the eigenvalues evolve from initial to final values without any tendency towards level-crossing. For $\Delta \bar{m}^{2}<0$, by contrast, the eigenvalues initially head towards each other as if to experience a level-crossing. However, whether this level-crossing actually occurs depends on the value of the mixing. For $\bar{\xi}>0$, the non-zero mixing between mass eigenstates induces a level repulsion which ultimately prevents a direct level-crossing. As a result the eigenvalues veer away from each other and ultimately assume the same late-time values that they had for $\Delta \bar{m}^{2}>0$. It is the transient behavior of the mass spectrum in Fig. 2 between these two endpoints that will play a subtle but important role in the results that follow.

\section{SURVEY OF PHENOMENA}

The total energy density is the quantity of central importance in the moduli problem, particularly at late times after the potential has been dynamically generated. In the mass-eigenstate basis this is given by

$$
\rho=\frac{1}{2} \sum_{\lambda}\left[\dot{\phi}_{\lambda}^{2}+\left(\lambda^{2}+\dot{\theta}^{2}\right) \phi_{\lambda}^{2}\right]+\dot{\theta} \sum_{\lambda \lambda^{\prime}} \dot{\phi}_{\lambda} \epsilon_{\lambda \lambda^{\prime}} \phi_{\lambda^{\prime}}
$$

where $\epsilon_{\lambda \lambda^{\prime}}$ is the Levi-Civita symbol. In different sectors of parameter space we will find that both strong enhancements and suppressions to $\bar{\rho}$ can occur. Furthermore, we are interested not only in the total late-time energy density but also in its distribution between the two individual components $\bar{\rho}_{\lambda}$ associated with the two moduli fields. These are the issues that we shall study in this section.

\section{The Total Energy Density}

The most complete way to survey the physics within this regime is to vary both $\tau_{G}$ and $\Delta_{G}$. We shall choose $\tau_{G}$ so as to avoid the situation in which neither field begins oscillating until after the phase transition has completed — a region which is uninteresting for our purposes.

Our results are shown in Fig. 3. In Fig. 3(a) and Fig. 3(b) we assume $\tau_{G}=1$ for radiation- and matter-dominated universes respectively, while in Fig. 3(c) we assume $\tau_{G} \gg 1$. In the latter regime matter- and radiation-dominated universes yield the same results. In each of these figures we plot values of the late-time mixing saturation $\bar{\xi}$ within the range $0 \leq \bar{\xi}<1$; note that we refrain from considering the actual limiting case with $\bar{\xi}=1$ when discussing late-time quantities such as the late-time energy density $\bar{\rho}$ because the lighter field $\phi_{\lambda_{0}}$ remains exactly massless at late times when $\bar{\xi}=1$. We avoid studying such a case since it implies the moduli potential was never stabilized.
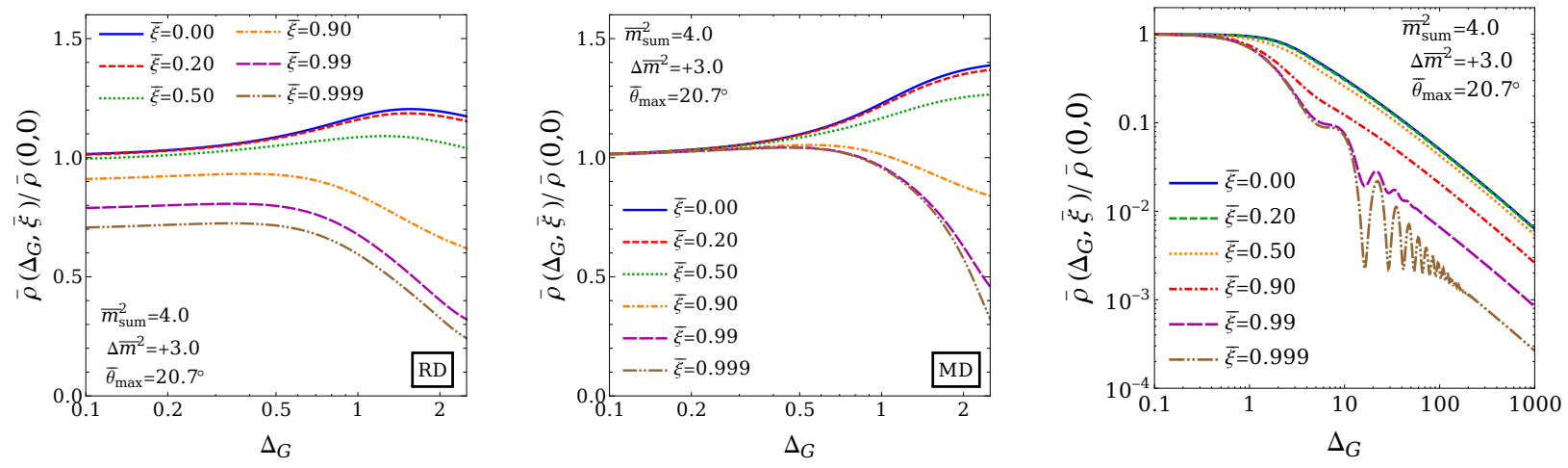

FIGURE 3. Behavior of the total late-time energy density $\bar{\rho}\left(\Delta_{G}, \bar{\xi}\right)$ when mixing effects, parametrized by $\bar{\xi}$, are combined with a non-zero width $\Delta_{G}$ for the mass-generating phase transition. In each case we plot the late-time energy density $\bar{\rho}$ as a function of the phase-transition width $\Delta_{G}$ for different values of the mixing saturation $\bar{\xi}$, where in each panel $\bar{\rho}$ is normalized to its value for $\Delta_{G}=0$ and $\bar{\xi}=0$. In the left and center panels a relatively small fiducial time $\tau_{G}=1.0$ is chosen so that the fields commence coherent oscillations near the time $\tau_{G}$, while in the right panel a large $\tau_{G}=10^{4}$ is chosen so that oscillations begin earlier in the phase transition. In the left and center panels we have assumed radiation- and matter-dominated universes, respectively, while in the right panel the results are insensitive to this choice. We have taken $\bar{m}_{\text {sum }}^{2}=4$ and $\Delta \bar{m}^{2}=+3$ in each panel. 
These figures illustrate the effects of assuming a finite width $\Delta_{G}$ for the mass-generating phase transition in conjunction with non-zero mixing between our two fields. For the small- $\tau_{G}$ regime plotted in Figs. 3(a) and 3(b), we observe that small mixing saturation enhances the late-time energy density $\bar{\rho}$. By contrast, we see that large mixing saturation suppresses the late-time energy density $\bar{\rho}$. Indeed, we see from Figs. 3(a) and 3(b) that these effects in both directions are more pronounced for matter-dominated universes than for radiation-dominated universes.

For the large- $\tau_{G}$ regime plotted in Fig. 3(c) all late-time energy densities $\bar{\rho}$, regardless of the mixing saturation $\bar{\xi}$, share a common value when $\Delta_{G}=0$. However, unlike the small- $\tau_{G}$ case, the late-time energy densities experience no enhancement at all, even in the absence of mixing $(\bar{\xi}=0)$. Indeed, $\bar{\rho}$ experiences only a suppression for non-zero $\Delta_{G}$ - a suppression which, given the logarithmic $\bar{\rho}$ axes within Fig. 3(c), grows much more severe than it was for small $\tau_{G}$. Finally, we observe the emergence of a non-monotonic "oscillatory" behavior for $\bar{\rho}$ as a function of $\Delta_{G}$ when $\tau_{G}$ is large and when the mixing saturation $\bar{\xi}$ grows close to 1 . These oscillations, which are actually resonant enhancements, will be discussed below, and indicate a strong sensitivity of the suppression factor relative to even small variations in the width $\Delta_{G}$.

All of these results illustrate the dramatic consequences that result when we consider not only a non-trivial time-dependence for moduli mass generation, but a non-trivial mixing as well. The enhancements and suppressions experienced by $\bar{\rho}$ are typically quite large, stretching from $20 \%$ or $30 \%$ in the case of enhancements all the way to many orders of magnitude in the case of suppressions. This demonstrates that the magnitude of the timescale over which the masses of the moduli are generated can have significant repercussions when mixing is fully considered. In particular, if the mixing between the moduli is near saturation and if the mass generation timescale exceeds $\Delta_{G} \gtrsim 2 \pi / \lambda_{1}$, the total energy density of the moduli can be hugely suppressed.

\section{Individual Component Energy Densities and Parametric Resonances}

We now turn to the distribution of this total energy density $\bar{\rho}$ between the two moduli. The decay rates of the two moduli vary, and depending on the mixing these rates could be quite different. In such cases, the individual abundances associated with each field separately become phenomenologically relevant.

In Fig. 4, we show the behavior of the individual late-time energy densities associated with our two moduli $\phi_{\lambda_{0}}$ and $\phi_{\lambda_{1}}$ for a mass-generating phase transition occurring at $\tau_{G}=10^{4}$. We first observe the unmixed $\xi \rightarrow 0$ limit, which corresponds to studying only a single field $\phi_{\lambda_{1}}$ (since the other field becomes massless and retains no amplitude). We observe from Fig. 4(b) that in this case the corresponding late-time energy density remains essentially constant (i.e., independent of $\Delta_{G}$ ) for $\Delta_{G} \lesssim 2 \pi / \lambda_{1}$. The effects of introducing a non-zero width for our phase transition will be essentially invisible if the mass generation occurs more rapidly than the natural timescale of oscillations of our (underdamped) field $\phi_{\lambda}$. However, for $\Delta_{G} \gtrsim 2 \pi / \lambda$, the field oscillations tend to compete against the process of mass generation. As a result, the mass-generating phase transition is less efficient in pumping energy density into our system, thereby inducing a suppression in the late-time energy $\bar{\rho}_{\lambda}$ which ultimately scales as an inverse power of $\Delta_{G}$.

Turning on a mixing between the two fields has a number of important effects on their individual late-time energy densities. For the lighter fields $\phi_{\lambda_{0}}$ [as considered in Fig. 4(a)], the most prominent effect is, of course, the set of very strong oscillations which are induced for mixing-saturated configurations $\bar{\xi} \lesssim 1$ and widths $1 \lesssim \Delta_{G} \lesssim 100$. We see that these oscillations are relatively large, occasionally enhancing the corresponding late-time energy density by several orders of magnitude!

These oscillations are a manifestation of a parametric resonance in the lighter field. This resonance emanates from the non-monotonic mass evolution that occurs in the lighter field as the mixing saturation is increased, a feature that is readily seen in Fig. 2. As the mixing becomes saturated, the mass evolution takes the form of a "pulse" near the phase transition. If the mass of the field (i.e. the "height" of the pulse) is close to a half-integer multiple of the effective frequency of the pulse, the field experiences a resonant enhancement. This is the effect responsible for the extremely large enhancements of $\bar{\rho}_{\lambda_{0}}$ seen in Fig. 4(a).

By contrast, for the heavier fields $\phi_{\lambda_{1}}$ [for which the corresponding energy density is plotted in Fig. 4(b)], the effects of non-zero mixing are quite the opposite: the heavier mass experiences no parametrically resonant enhancement, and instead there is now an additional suppression which helps to drive the corresponding energy densities to even smaller values, as functions of $\Delta_{G}$, than we had for the lighter fields! Unlike the lighter field $\phi_{\lambda_{0}}$, which obtains its energy density directly from the phase transition through the generation of a non-zero mass, the heavier field $\phi_{\lambda_{1}}$ actually acquires its energy density when it acquires an overall field amplitude through mixing with the lighter field. When the timescale of the field rotation is large compared to $2 \pi / \lambda_{1}$, the field amplitude cannot be generated efficiently and its energy density is reduced. 

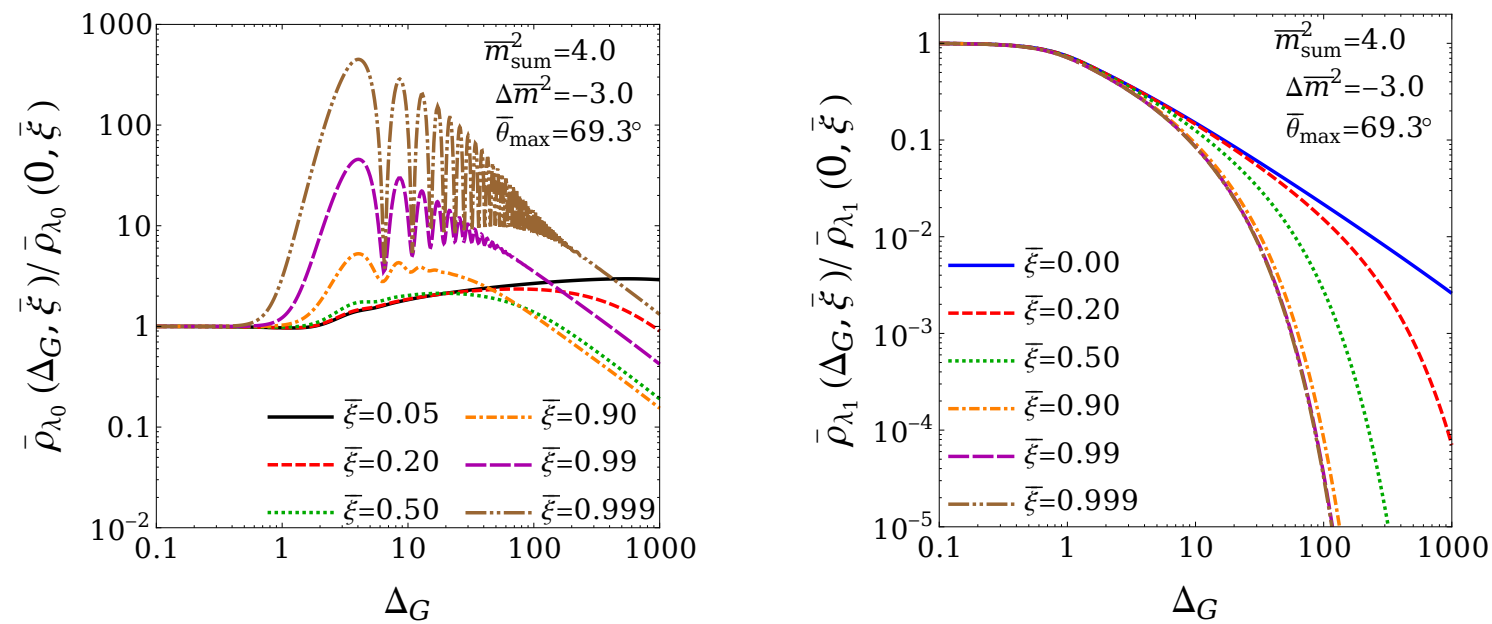

FIGURE 4. Behavior of the individual late-time energy density components $\bar{\rho}_{\lambda_{0,1}}\left(\Delta_{G}, \bar{\xi}\right)$ when mixing effects, parametrized by $\bar{\xi}$, are combined with a non-zero width $\Delta_{G}$ for the mass-generating phase transition. In these plots we adopt the large fiducial time $\tau_{G}=10^{4}$ assumed in Fig. 3(c), which leads to identical results for radiation- and matter-dominated universes. In the left panel we show results for $\bar{\rho}_{\lambda_{0}}$ (associated with the lighter state), while in the right panel we show results for $\bar{\rho}_{\lambda_{1}}$ (associated with the heavier state). We have taken $\bar{m}_{\text {sum }}^{2}=4$ and $\Delta \bar{m}^{2}=-3$. In each case we plot the late-time energy density $\bar{\rho}_{\lambda}$ as a function of the phase-transition width $\Delta_{G}$ for different values of the mixing saturation $\bar{\xi}$, normalized to its value for $\Delta_{G}=0$. Note that we refrain from plotting curves for $\bar{\xi}=0$ (and instead plot for $\bar{\xi}=0.05$ ) in cases where the corresponding energy densities vanish.

We are also interested in how the energy density is distributed between the two moduli fields after mass generation. Towards this end we shall study the fraction $\bar{\rho}_{\lambda_{0}} / \bar{\rho}$ of the total late-time energy density that the lighter field holds. In the $\Delta_{G} \rightarrow 0$ case of an instantaneous phase transition and in the $\lambda_{i} \tau_{G} \gg 1$ regime, this fraction is given by:

$$
\frac{\bar{\rho}_{\lambda_{0}}(0, \bar{\xi})}{\bar{\rho}(0, \bar{\xi})}=\frac{\bar{\lambda}_{0}^{2} \cos ^{2} \bar{\theta}}{\bar{\lambda}_{0}^{2} \cos ^{2} \bar{\theta}+\bar{\lambda}_{1}^{2} \sin ^{2} \bar{\theta}}=\cos ^{2} \bar{\theta}\left(\frac{1-\bar{\eta} \sec 2 \bar{\theta}}{1-\bar{\eta}}\right) .
$$

As $\Delta_{G}$ grows larger, this ratio will change. For the parameter set in Fig. 3(c), the corresponding fractional results are shown in Fig. 5 as a function of $\Delta_{G}$. It is easy to understand the behavior shown in Fig. 5. Regardless of the fraction of the total late-time energy density associated with the lighter field $\phi_{\lambda_{0}}$ when $\Delta_{G}=0$, we have already seen
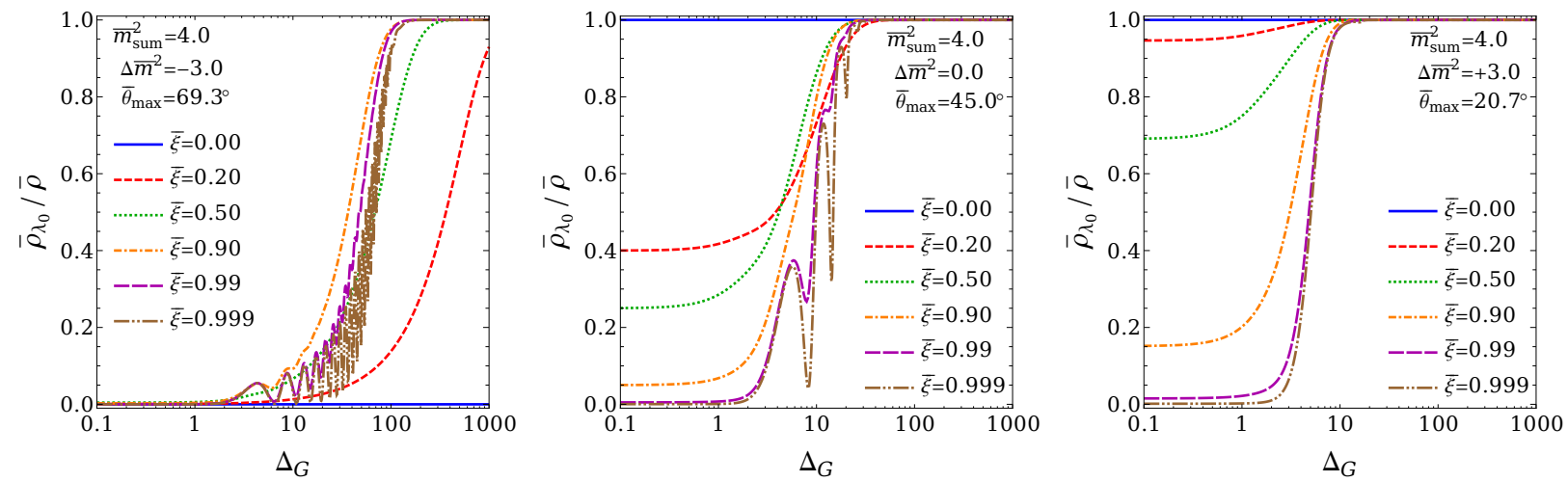

FIGURE 5. The fraction of the total late-time energy density $\bar{\rho}\left(\Delta_{G}, \xi\right)$ which is associated with the lighter field $\phi_{\lambda_{0}}$, plotted as functions of $\Delta_{G}$ for different values of $\xi$, with $\tau_{G}=10^{4}$. Regardless of the small- $\Delta_{G}$ values of this fraction [as given analytically in Eq. (16)], we see that increasing the width $\Delta_{G}$ of the phase transition generally has the net effect of transferring an increasing amount of the total energy density into the lighter field. Thus, simply by adjusting the width of the phase transition, we see that we can often entirely reverse the distribution of the late-time energy density. 

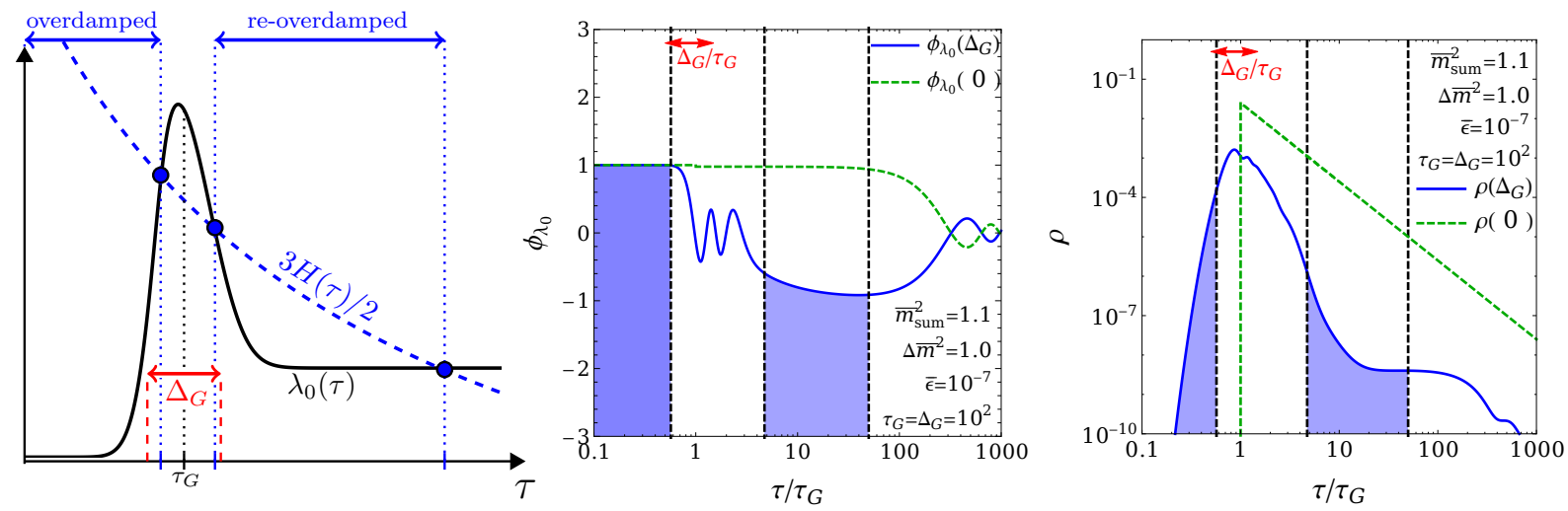

FIGURE 6. Left panel: A schematic illustrating the "pulse"-like behavior that the lower-mass eigenvalue $\lambda_{0}(\tau)$ can develop in highly mixing-saturated $(\epsilon \equiv 1-\xi \rightarrow 0)$ regimes. The corresponding moduli field $\phi_{\lambda_{0}}$ therefore experiences not just the usual transition from overdamped to underdamped behavior, but also a novel re-overdamping transition. As shown, this re-overdamped phase can persist for considerably longer than the width $\Delta_{G}$ of the pulse, and thus can have a significant impact on the resulting cosmological history. Center and right panels: The behavior of the lighter field $\phi_{\lambda_{0}}$ (center panel) and the total moduli energy density $\rho$ (right panel) in a situation exhibiting re-overdamping. The fields and corresponding energy densities are normalized so that $\phi_{\lambda_{0}}=1$ for $\tau \ll \tau_{G}$. Time intervals during which the system is overdamped are shaded (blue), while the width $\Delta_{G}$ of the original mass-generating phase transition is also indicated (red). Superimposed for comparison on these panels are the values that our system would have exhibited in the $\Delta_{G} \rightarrow 0$ limit (green). We thus see that the re-overdamped phase can easily persist throughout a large period of cosmological history, with a phenomenology that is quite different from those of the traditional overdamped or underdamped phases.

that the energy densities associated with the heavier fields are typically more suppressed as a function of $\Delta_{G}$ than are the energy densities associated with the lighter fields. Thus, regardless of the late-time energy-density configuration when the phase transition is instantaneous, we see that increasing the width of the phase transition has the effect of throwing an increasingly large share of the total late-time energy density into the lighter field. Indeed, in some cases, we see that we can entirely reverse the distribution of the total energy density from the heavier field to the lighter field, simply by adjusting the timescale over which the phase transition occurs! In models where the potentials for moduli are generated over timescales $\Delta_{G} \gtrsim 2 \pi / \lambda_{1}$, sufficiently large mixing can thus allow the energy density to be dumped into the faster-decaying heavier field.

\section{Re-Overdamping}

As we have seen, any scalar field $\phi$ with mass $\lambda$ experiences overdamped behavior (and thus functions as vacuum energy) at times $\tau$ for which $3 H / 2 \gtrsim \lambda$. By contrast, such a field experiences underdamped behavior (and thus functions as matter) when $3 H / 2 \lesssim \lambda$. Of course, $H \sim 1 / \tau$ in any post-inflationary epoch. We saw in the previous subsection that the non-monotonic time-dependence of $\lambda_{0}$ in a case with highly saturated mixing can produce parametric resonances in the lighter field. However, this non-monotonic time-dependence can also produce a second phenomenon, one which we refer to as "re-overdamping".

To see this, let us consider the situation that can occur with parameter configurations that are highly mixingsaturated, with $\bar{\epsilon} \equiv 1-\bar{\xi} \rightarrow 0$. As illustrated in Fig. 6(a), the "pulse" developed in the mass $\lambda_{0}$ leads to parameter configurations for which the $3 H(\tau) / 2$ curve may cross the $\lambda(\tau)$ curve not just once but three times, resulting in a field which is overdamped for early times, then becomes underdamped, but then experiences a re-overdamped phase before eventually settling back into a final underdamped state. The period during which the field exists in a re-overdamped phase can be of extremely long duration - even relative to the width of the phase transition that produced it.

This re-overdamped phase of the theory should not be associated with vacuum energy. Indeed, at the moment of transition into the re-overdamped phase, oscillatory behavior ceases but our field value re-enters the overdamped phase carrying a non-zero velocity $\dot{\phi}$. In this sense, then, the re-overdamped phase represents a truly different behavior for our field values - one which is neither vacuum energy (constant field values) nor matter (oscillatory field values), but something completely different which transcends our traditional expectations for moduli in an expanding universe. 
Further details concerning all of these phenomena, along with their implications in a variety of settings, are discussed in Ref. [1].

\section{ACKNOWLEDGMENTS}

KRD and BT would like to thank CETUP* for hospitality during the 2015 Dark-Matter Workshop and PPC Conference. BT would also like to thank CETUP* for financial support during the workshop. The research activities of KRD and JK were supported in part by the Department of Energy under Grant DE-FG02-13ER-41976, while the research activities of BT were supported in part by internal funding from Reed College. The research activities of KRD were also supported in part by the National Science Foundation through its employee IR/D program. The opinions and conclusions expressed herein are those of the authors, and do not represent any funding agencies.

\section{REFERENCES}

[1] K. R. Dienes, J. Kost, and B. Thomas. arXiv:1509.00470 (September 2015), to appear in Phys. Rev. D.

[2] T. Banks, D. B. Kaplan, and A E. Nelson. Phys. Rev., D49:779-787, 1994.

[3] B. de Carlos, J.A. Casas, F. Quevedo, and E. Roulet. Phys. Lett., B318:447-456, 1993.

[4] T. Banks, M. Berkooz, and P. J. Steinhardt. Phys. Rev., D52:705-716, 1995.

[5] G.D. Coughlan, W. Fischler, E. W. Kolb, S. Raby, and G. G. Ross. Phys. Lett., B131:59, 1983.

[6] A. D. Linde. Phys. Rev., D53:4129-4132, 1996.

[7] K. R. Dienes, E. Dudas, and T. Gherghetta. Phys. Rev., D62:105023, 2000.

[8] K. R. Dienes and B. Thomas. Phys. Rev., D85:083523, 2012.

[9] K. R. Dienes and B. Thomas. Phys. Rev., D85:083524, 2012.

[10] K.S. Babu, S. M. Barr, and D. Seckel. Phys. Lett., B336:213-220, 1994. 